\title{
Двухтоновые нелинейные электростатические волны в квантовой электронно-дырочной плазме полупроводников
}

\author{
(C) A.Е. Дубинов ${ }^{1,2,3}$, И.Н. Китаев ${ }^{1,2,3}$ \\ ${ }^{1}$ Российский федеральный ядерный центр - \\ Всероссийский научно-исследовательский институт экспериментальной фиизики, \\ 607188 Саров, Россия \\ ${ }^{2}$ Национальный исследовательский ядерный университет „МИФИ“ (НИЯУ МИФИ), \\ 115409 Москва, Россия \\ ${ }^{3}$ Саровский фризико-технический институт \\ Национального исследовательского ядерного университета „МИФИ“, \\ 607186 Саров, Россия \\ E-mail: dubinov-ae@yandex.ru
}

(Получена 24 февраля 2015 г. Принята к печати 13 апреля 2016 г.)

\begin{abstract}
Рассмотрены продольные электростатические волны в квантовой электронно-дырочной плазме полупроводников с учетом вырожденности электронов и дырок, а также с учетом обменного взаимодействия. В рамках линейной теории найдено, что дисперсионная кривая продольных волн имеет две ветви плазмонную и фононную. Найдено выражение для критической частоты отсечки для плазменных колебаний, а также выражение для скорости звука для акустических колебаний. Показано, что плазменная волна всегда существует в виде суперпозиции двух составляющих, имеющих разный период и длину волны. В рамках нелинейной теории получены два нелинейных решения, одно из которых имеет форму простой суперпозиции двух тонов, а другое - имеет форму биений.
\end{abstract}

DOI: 10.21883/FTP.2017.01.43989.7871

\section{1. Введение}

Коллективные волновые процессы в квантовой электронно-дырочной плазме (eh-плазме) полупроводников давно привлекают внимание специалистов [1-5]. Для их понимания и теоретического описания часто применяют идеи и подходы, развитые для обычной газообразной плазмы [6-9]. Эта аналогия позволила обнаружить в полупроводниках и описать многие волновые явления, известные в обычной плазме, например, электростатические и циклотронные волны.

Один из эффективных подходов для описания волн в $e h$-плазме является метод на основе гидродинамических уравнений [10]. Гидродинамические модели волн в $e h$-плазме постепенно усложнялись и совершенствовались путем учета вырожденности электронов и дырок, квантовой силы Бома, а также обменных потенциалов. Развитие этих моделей можно проследить по работам $[4,11-16]$.

Недавно в работе [17] был предложен подход на основе гидродинамического описания электростатических волн в квантовой плазме, позволяющий получать нелинейные решения уравнений. В рамках этого подхода были проанализированы нелинейные ленгмюровские волны в квантовой плазме и показано, что эти волны всегда двухцветны. При этом низкочастотная составляющая волны соответствует классическим ленгмюровским волнам, а высокочастотная составляющая - свободным квантовым колебаниям электронов. Фактически в работе [17] была рассмотрена динамика одной компоненты плазмы - электронной, в то время как ионы считались достаточно массивными и неподвижными.

В данной работе рассматриваются продольные электростатические волны с помощью подхода, предложенного в работе [17], который обобщен на случай $e h$-плазмы с двумя видами подвижных компонент электронов и дырок. Кроме того, учтен обменный потенциал так, как это сделано в работе [4]. Далее, сначала в рамках линейной теории выведено и проанализировано дисперсионное уравнение, а затем представлены решения нелинейных уравнений и их интерпретация.

\section{2. Линейная теория}

Будем рассматривать квазинейтральную бесстолкновительную $e h$-плазму, в которой электроны и дырки имеют, вообще говоря, разные эффективные массы. При этом электроны и дырки подчиняются уравнениям состояния вырожденного ферми-газа:

$$
p_{e, h}=\frac{\left(3 \pi^{2}\right)^{2 / 3}}{5} \frac{\hbar^{2}}{m_{e, h}} n_{e, h}^{5 / 3},
$$

где $m_{e, h}$ - эффективные массы электронов и дырок, $n_{e, h}$ - концентрации электронных и дырочных компонент в $e h$-плазме, $p_{e, h}$ - давления в них.

Представление о бесстолкновительной плазме применимо к рассматриваемой здесь модели, так как вырожденный ферми-газ обладает своеобразным свойством с увеличением его плотности он становится все более и более идеальным [18]. 
Будем исходить из следующих одномерных уравнений:

$$
\begin{gathered}
\frac{\partial n_{e, h}}{\partial t}+\frac{\partial}{\partial z}\left(n_{e, h} u_{e h}\right)=0 \\
\frac{\partial u_{e, h}}{\partial t}+u_{e, h} \frac{\partial u_{e, h}}{\partial z}= \pm \frac{e}{m_{e, h}} \frac{\partial \varphi}{\partial z}-\frac{1}{m_{e, h} n_{e, h}} \frac{\partial p_{e, h}}{\partial z}+\frac{\hbar^{2}}{4 m_{e, h}^{2}} \\
\times \frac{\partial}{\partial z}\left\{\frac{1}{n_{e, h}}\left[\frac{\partial^{2} n_{e, h}}{\partial z^{2}}-\frac{1}{2 n_{e, h}}\left(\frac{\partial n_{e, h}}{\partial z}\right)^{2}\right]\right\}+\frac{1}{m_{e, h}} \frac{\partial V_{x c e, x c h}}{\partial z}, \\
\frac{\partial^{2} \varphi}{\partial z^{2}}=4 \pi e\left(n_{e}-n_{h}\right) .
\end{gathered}
$$

Два уравнения (2) представляют собой уравнения непрерывности для электронов и дырок соответственно, два уравнения (3) - уравнения их движения и (4) уравнение Пуассона. В них обозначено: $u_{e, h}-$ скорости электронов и дырок, $\varphi-$ электростатический потенциал, $V_{x c e, x c h}$ - обменный потенциал для электронов и дырок, $e-$ элементарный электрический заряд, $\hbar-$ постоянная Планка. В правой части уравнения (3) знак плюс соответствует электронам, а минус - дыркам.

В правой части уравнения (3) имеются четыре слагаемых, которые представляют собой электрическую силу, газодинамическую силу, квантовую силу Бома и эффективную обменную силу соответственно.

Согласно работам $[4,19]$, можно использовать обменные потенциалы $V_{x c e, x c h}$, записанные в виде

$$
V_{x c e, x c h}=-\frac{a_{1} e^{2} n_{e, h}^{1 / 3}}{\varepsilon_{L}}\left[1+\frac{a_{2} n_{e, h}^{-1 / 3}}{a_{e, h}^{*}} \ln \left(1+a_{3} a_{e, h}^{*} n_{e, h}^{1 / 3}\right)\right],
$$

где обозначено $a_{e, h}^{*}=\frac{\varepsilon_{L} \hbar^{2}}{m_{e, h} e^{2}}, \quad \varepsilon_{L}-$ линейная диэлектрическая постоянная полупроводника, $a_{1}=0.985$, $a_{2}=0.034, a_{3}=18.37$.

Пусть вдоль направления $0 z$ распространяется плоская гармоническая волна небольшой амплитуды, в которой физические величины изменяются по закону

$$
\left\{\begin{array}{l}
n_{e, h}=n_{0}+\bar{n}_{e, h} \exp [i(k z-\omega t)], \\
u_{e, h}=\bar{u}_{e, h} \exp [i(k z-\omega t)], \\
\varphi=\bar{\varphi} \exp [i(k z-\omega t)] .
\end{array}\right.
$$

Амплитуды волнового возмущения плазмы в (6) отмечены черточкой сверху и считаются малыми. Подставляя (6) в исходные уравнения (2)-(4) и оставляя только линейные по малым амплитудам члены, после несложных алгебраических преобразований получим дисперсионное уравнение в виде

$$
\begin{aligned}
& \left(\omega^{2}-\omega_{p e}^{2}-\frac{V_{\mathrm{Fe}}^{2}}{3} k^{2}-A_{e} k^{2}-\frac{\hbar^{2} k^{4}}{4 m_{e}^{2}}\right) \\
& \quad \times\left(\omega^{2}-\omega_{p h}^{2}-\frac{V_{\mathrm{Fh}}^{2}}{3} k^{2}-A_{h} k^{2}-\frac{\hbar^{2} k^{4}}{4 m_{h}^{2}}\right)=\omega_{p e}^{2} \omega_{p h}^{2},
\end{aligned}
$$

в котором применены следующие обозначения:

$$
\omega_{p e, p h}^{2}=\frac{4 \pi e^{2} n_{0}}{m_{e, h}}
$$

— плазменные частоты электронов и дырок,

$$
V_{\mathrm{F} e, \mathrm{~F} h}=\left(3 \pi^{2}\right)^{1 / 3} \frac{\hbar}{m_{e, h}} n_{0}^{1 / 3}
$$

- скорости Ферми,

$$
A_{e, h}=\frac{a_{1} e^{2} n_{0}^{1 / 3}}{3 m_{e, h} \varepsilon_{L}}\left(1+\frac{a_{2} a_{3}}{1+a_{3} a_{e, h}^{*} n_{0}^{1 / 3}}\right)
$$

- обменные коэффициенты, имеющие размерность квадрата скорости.

Если разрешить дисперсионное уравнение (7) относительно частоты, то можно убедиться, что дисперсионная зависимость содержит две ветви

$$
\begin{aligned}
\omega^{2}= & \frac{1}{2}\left(\omega_{p e}^{2}+\omega_{p h}^{2}+\frac{V_{\mathrm{Fe}}^{2}}{3} k^{2}+A_{e} k^{2}+A_{h} k^{2}\right. \\
& \left.+\frac{V_{\mathrm{Fh}}^{2}}{3} k^{2}+\frac{\hbar^{2} k^{4}}{4 m_{e}^{2}}+\frac{\hbar^{2} k^{4}}{4 m_{h}^{2}} \pm \sqrt{\Delta}\right)
\end{aligned}
$$

с дискриминантом

$$
\begin{aligned}
\Delta= & \left(\omega_{p e}^{2}-\omega_{p h}^{2}+\frac{V_{\mathrm{Fe}}^{2}}{3} k^{2}-\frac{V_{\mathrm{Fh}}^{2}}{3} k^{2}+A_{e} k^{2}-A_{h} k^{2}\right. \\
& \left.+\frac{\hbar^{2} k^{4}}{4 m_{e}^{2}}-\frac{\hbar^{2} k^{4}}{4 m_{h}^{2}}\right)^{2}+4 \omega_{p e}^{2} \omega_{p h}^{2} .
\end{aligned}
$$

В зависимости (8) знак плюс перед квадратным корнем соответствует оптической (плазмонной) ветви, для которой электроны и дырки колеблются в волне противофазно, а знак минус - акустической (фононной) ветви, для которой электроны и дырки колеблются в волне синфазно.

Можно получить выражение для скорости звука для акустической ветви

$$
c_{s}=\lim _{k \rightarrow 0} \frac{\omega}{k}=\sqrt{\frac{2}{3} \frac{\omega_{p e}^{2} V_{\mathrm{Fh}}^{2}+\omega_{p h}^{2} V_{\mathrm{Fe}}^{2}+3 \omega_{p e}^{2} A_{h}+3 \omega_{p h}^{2} A_{e}}{\omega_{p e}^{2}+\omega_{p h}^{2}}} .
$$

На рис. 1 построен график дисперсионной зависимости (8). На графике видно, что обе ветви очень круто, пропорционально $k^{2}$ уходят вверх благодаря квантовой силе Бома, а роль обменного взаимодействия при этом невелика: оно приводит лишь к изменению коэффициента линейного по $k$ слагаемого. Таким образом, здесь найдено важное отличие квантовой $e h$-плазмы (рост $\sim k^{2}$ ) от классической плазмы, для которой кривые уходят вверх, приближаясь к асимптоте только $\sim k$.

И если для классической плазмы возможно лишь однократное пересечение ветвей прямым лучом, выходящим из начала координат, то в случае квантовой плазмы любая ветвь пересекается лучом двукратно. Физически это соответствует тому, что как для плазмонной ветви, 
так и для фононной ветви любая гармоническая волна всегда имеет два тона, которые бегут с одинаковыми фазовыми скоростями.

На рис. 1 показаны два луча, каждому из которых соответствует своя фазовая скорость. Видно, что оба луча пересекают плазмонную кривую дважды. При этом лучу 1 будет соответствовать суперпозиция двух тонов, сильно отличающихся друг от друга по частоте и длине волны. Для луча 2 точки пересечения близки друг другу, следовательно, волна, бегущая с соответствующей фазовой скоростью, должна иметь форму биений.

\section{3. Нелинейная теория}

Вернемся к уравнениям (2)-(4). Перейдем к новой переменной: $\xi=z-V t$, предполагая, что все искомые функции зависят только от нее. Тогда исходная система уравнений запишется в нормированном виде следующим образом:

$$
\begin{gathered}
-V \frac{\partial n_{e}, h}{\partial \xi}+\frac{\partial}{\partial \xi}\left(n_{e, h} u_{e, h}\right)=0 \\
-V \frac{\partial u_{e}}{\partial \xi}+u_{e} \frac{\partial u_{e}}{\partial \xi}=\frac{\partial \varphi}{\partial \xi}-\frac{1}{3} n_{e}^{-1 / 3} \frac{\partial n_{e}}{\partial \xi}+\frac{H_{e}^{2}}{4} \\
\times \frac{\partial}{\partial \xi}\left\{\frac{1}{n_{e}}\left[\frac{\partial^{2} n_{e}}{\partial \xi^{2}}-\frac{1}{2 n_{e}}\left(\frac{\partial n_{e}}{\partial \xi}\right)^{2}\right]\right\}-\frac{a_{1}}{\left(3 \pi^{2}\right)^{2 / 3} a_{e}^{*} n_{0}^{1 / 3}} \\
\times \frac{\partial}{\partial \xi}\left\{n_{e}^{1 / 3}\left[1+\frac{a_{2} n_{e}^{-1 / 3}}{a_{e}^{*} n_{0}^{1 / 3}} \ln \left(1+a_{3} a_{e}^{*} n_{0}^{1 / 3} n_{e}^{1 / 3}\right)\right]\right\}, \\
-V \frac{\partial u_{h}}{\partial \xi}+u_{h} \frac{\partial u_{h}}{\partial \xi}=-M \frac{\partial \varphi}{\partial \xi}-\frac{M^{2}}{3} n_{h}^{-1 / 3} \frac{\partial n_{h}}{\partial \xi} \\
+M^{2} \frac{H_{e}^{2}}{4} \frac{\partial}{\partial \xi}\left\{\frac{1}{n_{h}}\left[\frac{\partial^{2} n_{h}}{\partial \xi^{2}}-\frac{1}{2 n_{h}}\left(\frac{\partial n_{h}}{\partial \xi}\right)^{2}\right]\right\}-\frac{a_{1} M}{\left(3 \pi^{2}\right)^{2 / 3} a_{e}^{*} n_{0}^{1 / 3}} \\
\times \frac{\partial}{\partial \xi}\left\{n_{h}^{1 / 3}\left[1+\frac{a_{2} n_{h}^{-1 / 3}}{M a_{e}^{*} n_{0}^{1 / 3}} \ln \left(1+a_{3} M a_{e}^{*} n_{0}^{1 / 3} n_{h}^{1 / 3}\right)\right]\right\} \\
\frac{\partial^{2} \varphi}{\partial \xi^{2}}=n_{e}-n_{p},
\end{gathered}
$$

где все концентрации нормированы на $n_{0}$, все скорости - на $V_{\mathrm{Fe}}$, потенциал - на $\frac{m V_{\mathrm{Fe}}^{2}}{e}$ и обозначено $H_{e}=\frac{\hbar \omega_{p e}}{m V_{\mathrm{Fe}}^{2}}, M=\frac{m_{e}}{m_{h}}$.

Систему обыкновенных дифференциальных уравнений (11)-(14) можно решить численно, например, методом Рунге-Кутты. Приведем два характерных примера численного решения системы при различных значениях скорости $V$, соответствующих лучам 1 и 2 на рис. 1 .

Расчеты проводились при следующих начальных условиях: $\varphi(0)=0, \varphi^{\prime}(0)=0.15, \quad u_{e, h}(0)=0, \quad n_{e, h}(0)=1$, $n_{e}^{\prime}(0)=0.0325, \quad n_{h}^{\prime}(0)=0.03, \quad n_{e, h}^{\prime \prime}(0)=0 \quad$ и при следующих значениях параметров: $H=1.5, a_{e}^{*} n_{0}^{1 / 3}=1.5$, $M=0.7, V=1.79106$ для биений и $V=3$ для суперпозиции.
Решения представлены на рис. 2 и 3 . Для луча 1 волна, как и было выше предсказано линейной теорией, имеет форму простой суперпозиции двух тонов, а для луча $2-$ имеет форму биений.

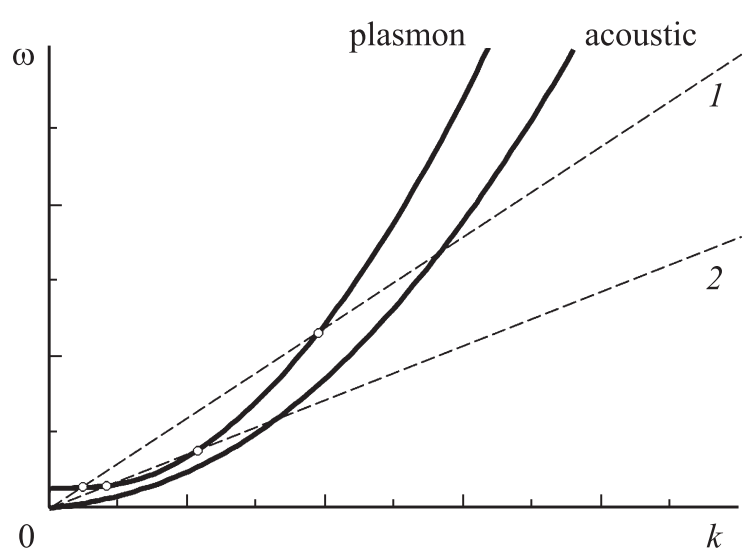

Рис. 1. График дисперсионной кривой: плазмонная ветвь помечена „plasmon“, фононная ветвь помечена „acoustic“; 1, 2номера лучей.
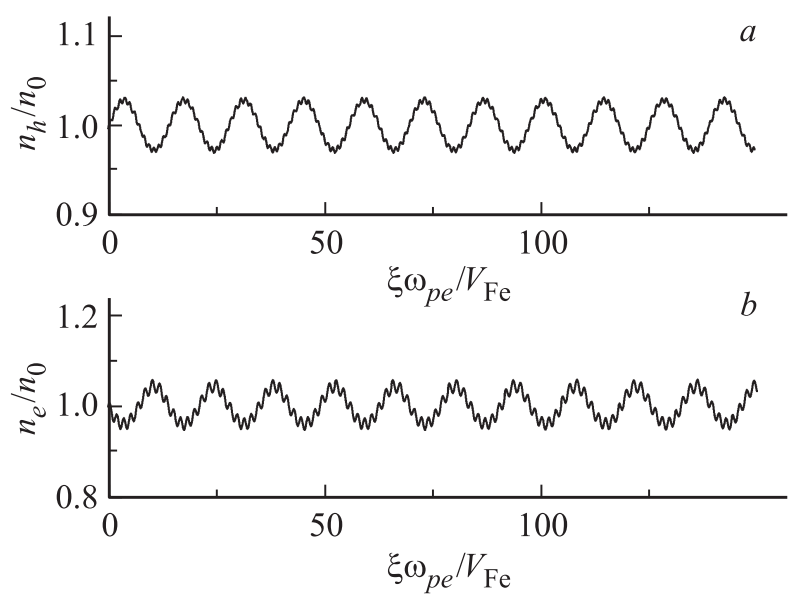

Рис. 2. Графики профилей концентрации дырок $(a)$ и электронов $(b)$ в плазменной волне, соответствующие простой суперпозиции тонов.
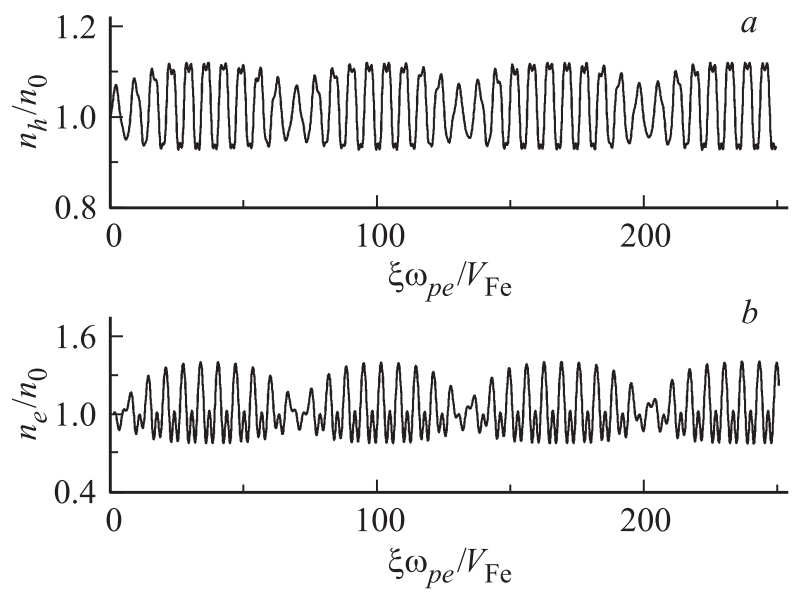

Рис. 3. Графики профилей концентрации дырок $(a)$ и электронов $(b)$ в плазменной волне, соответствующие биениям тонов. 
Параметры теллурида ртути при температуре $4.2 \mathrm{~K}$

\begin{tabular}{c|c}
\hline Параметры & HgTe \\
\hline$n_{0 e}, \mathrm{~cm}^{-3}$ & $8.5 \cdot 10^{14}$ \\
$m_{e}^{*}$ & $0.032 m_{e}$ \\
$m_{h}^{*}$ & $0.35 m_{h}$ \\
$\mu_{e}, \mathrm{~cm}^{2} / \mathrm{B} \cdot \mathrm{c}$ & $7.86 \cdot 10^{5}$ \\
$\varepsilon_{L}$ & 20.8 \\
$a_{\mathrm{B} e}^{*}=\frac{\varepsilon_{L} \hbar^{2}}{m_{e}^{*} e^{2}}, \AA$ & 343.97 \\
$a_{\mathrm{B} h}^{*}=\frac{\varepsilon_{L} \hbar^{2}}{m_{h}^{*} e^{2}}, \AA$ & 31.45 \\
$\tau_{e e} \approx \frac{m_{e}^{2} e^{2}}{n_{0 e} \hbar^{3}}, \mathrm{~ms}$ & 249.4
\end{tabular}

Проведенные исследования предсказывают существование подобных волн в квантово-вырожденной плазме полупроводников. В качестве примера полупроводникового материала, где они могли бы наблюдаться, можно указать теллурид ртути (HgTe) при низкой температуре, в котором электронно-дырочная плазма вырождена [20]. В таблице приведены некоторые полезные параметры для этого полупроводника при температуре $4.2 \mathrm{~K}$, взятые из [20] и позволяющие рассчитывать характеристики волн. В ней обозначено: $n_{0 e}-$ невозмущенная концентрация свободных электронов, $m_{e}^{*}, m_{h}^{*}$ - эффективные массы электронов и дырок, $\mu_{e}$ - подвижность электронов, $a_{\mathrm{B} e}^{*}, a_{\mathrm{B} h}^{*}-$ эффективные боровские радиусы электронов и дырок, $\tau_{e e}$ - время релаксации за счет электрон-электронного взаимодействия, $\varepsilon_{L}-$ линейная диэлектрическая постоянная полупроводника.

\section{4. Заключение}

Таким образом, в работе рассмотрены продольные электростатические волны в квантовой eh-плазме полупроводников с учетом вырожденности электронов и дырок, а также с учетом обменного взаимодействия.

В рамках линейной теории найдено, что дисперсионная кривая продольных волн имеет две ветви плазмонную и фононную. Найдено выражение для критической частоты отсечки для плазменных колебаний, а также выражение для скорости звука для акустических колебаний. Показано, что плазменная волна всегда существует в виде суперпозиции двух составляющих, имеющих разный период и длину волны.

В рамках нелинейной теории получены два нелинейных решения, одно из которых имеет форму простой суперпозиции двух тонов, а другое имеет форму биений.

\section{Список литературы}

[1] W.-P. Hong, Y.-D. Jung. Phys. Lett. A, 380, 131306 (2016).

[2] J. Furdyna. Phys. Rev. Lett., 16 (15), 646 (1966).

[3] С.Е. Кумеков. ФТП, 42 (8), 938 (2008).

[4] R.M. Amin. Physica Scripta, 90 (1), 015601 (2015).
[5] H. Haug, S.W. Koch. Quantum theory of the optical and electronic properties of semiconductors. (World Scientific Publishing Co. Pte. Ltd-Singapore, 2004).

[6] Ф. Платцман, П. Вольф. Волны и взаимодействия в плазме твердого тела (М., Мир, 1975).

[7] Ф.Г. Басс, Ю.Г. Гуревич. Горячие электроны и сильные электромагнитные волны в плазме полупроводников $и$ газового разряда (М., Наука, 1975).

[8] Ю.К. Пожела. Плазма и токовые неустойчивости в полупроводниках (М., Наука, 1977).

[9] Н.Н. Белецкий, А.А. Булгаков, С.И. Ханкина, В.М. Яковенко. Плазменные неустойчивости и нелинейные явления в полупроводниках (Киев, Наук. думка, 1984).

[10] P.A. Markowich, C.A. Ringhofer, C. Schmeiser. Semiconductor equations (Springer Verlag, Wien-N. Y., 1990).

[11] J.-R. Zhou, D.K. Ferry. IEEE Trans. Electron Dev., 40 (2), 421 (1993).

[12] C.L. Gardner. SIAM J. Appl. Math., 54 (2), 409 (1994).

[13] I. Gasser. Appl. Math. Lett., 14 (3), 279 (2001).

[14] G. Manfredi, F. Haas. Phys. Rev. B, 64 (7), 075316 (2001).

[15] A.E. Dubinov. Russian Microelectron., 30 (5), 339 (2001).

[16] M. Akbari-Moghanjoughi, P.K. Shukla. Phys. Rev. E, 86 (6), 066401 (2012).

[17] A.E. Dubinov, I.N. Kitaev. Phys. Plasmas., 21 (10), 102105 (2014).

[18] Л.Д. Ландау, Е.М. Лифшиц. Статистическая физика (М., Наука, 1995).

[19] F. Stern, S.D. Sarma. Phys. Rev. B, 30 (2), 840 (1984).

[20] М.И. Даунов, И.К. Камилов, С.Ф. Габибов. ФТП, 43 (2), 180 (2009).

Редактор А.Н. Смирнов

\section{Two-tone non-linear electrostatic waves in quantum electron-hole plasma of semiconductors}

A.E. Dubinov ${ }^{\mathbf{1 , 2 , 3}}$, I.N. Kitayev ${ }^{\mathbf{1 , 2 , 3}}$

${ }^{1}$ Russian Federal Nuclear Center -

All-Russia Scientific and Research Institute

of Experimental Physics (RFNC-VNIIEF),

607188 Sarov, Russia

${ }^{2}$ National Research Nuclear University „MEPhl“, 115409 Moscow, Russia

${ }^{3}$ Sarov State Institute of Physics and Technology (SarFTI)

of National Research Nuclear University MEPhl, 607186 Sarov, Russia

Abstract This paper considers longitudinal electrostatic waves in electron-hole plasma of semiconductors taking into account degeneration of the electrons and holes, and exchange interaction. In the frameworks of the linear theory it was found that dispersion curve of the longitudinal waves has two branches-plasmon and acoustic ones. An expression for the critical cutoff frequency for plasma oscillations and expression for sound speed of acoustic oscillations are found. The authors show that the plasma wave always exists in the form of superposition of two components. These components have different period and wavelength. Two non-linear solutions are obtained in the frameworks of non-linear theory. One solution has a form of simple superposition of two tones; another - the form of beats. 